3. - Linear operators in the theory of partial differential equations, Trans. Amer. Math. Soc. vol. 53 (1943) pp. 130-155.

4. ——, Two dimensional transonic flow patterns, Amer. J. Math. vol. 70 (1948) pp. 956-891.

5. E. Holmgren, Sur un problème aux limites pour l'equation $y^{m} \partial^{2} z / \partial x^{2}+\partial^{2} z / \partial y^{2}=0$, Arkiv for Mathematik, Astronomi och Fysik vol. 19, B (1927) pp. 1-3.

6. F. Tricomi, Sulle equazioni lineari alle derivate parzialo di $2^{\circ}$ ordine di tipo misto, Memorie della R. Accademia dei Lincei vol. 14 (1923) pp. 133-247.

HARVARD UNIVERSITY

\title{
AN INVERSION FORMULA FOR THE GENERALIZED STIELTJES TRANSFORM
}

D. B. SUMNER ${ }^{1}$

1. Introduction. The problem of finding formulae to invert the Stieltjes transforms

$$
\begin{aligned}
& f(x)=\int_{0}^{\infty} d \alpha(t) /(x+t), \\
& f(x)=\int_{0}^{\infty} \phi(t) d t /(x+t),
\end{aligned}
$$

and the generalized transforms

$$
\begin{aligned}
& f(x)=\int_{0}^{\infty} d \alpha(t) /(x+t)^{\rho}, \\
& f(x)=\int_{0}^{\infty} \phi(t) d t /(x+t)^{\rho},
\end{aligned}
$$

has been solved by Widder [A, pp. 7-60 $]^{2}$ and by Pollard [F, pp. 1416]. The function $\phi(t) \in L(0, \infty), \alpha(t)$ is a normalized function of

Received by the editors January 19, 1948, and, in revised form, February 26, 1948.

1 The subject matter of this paper forms part of a dissertation presented to the University of the Witwatersrand. The author wishes to acknowledge with gratitude facilities granted by the Principal and Council of that University, and assistance subsequently given by the Council for Scientific and Industrial Research of the Union of South Africa. In particular, the author acknowledges his indebtedness to Dr. J. P. Dalton, Emeritus Professor in Mathematics of the University of the Witwatersrand.

2 Roman letters in brackets refer to the bibliography at the end of the paper. 
bounded variation in $(0, R)$ for all positive $R$, the integrals converge for some complex $x$ which is neither zero nor negative, and $\rho$ is positive. Widder showed that if

$$
L_{k t}(f)=\frac{(-)^{k-1}}{k !(k-2) !}\left[t^{2 k-1} f^{(k-1)}(t)\right]^{(k)}
$$

then for (2), $\phi(t)=\lim _{k \rightarrow \infty} L_{k t}(f)$ at all points of the Lebesgue set for $\phi(t)$; and for (1), $\lim _{k \rightarrow \infty} \int_{0}^{t} L_{k u}(f) d u=\alpha(t)-\alpha(0+)$. Pollard defined the operator

$$
\left.L_{k t}^{\rho}(f)=\frac{(-)^{k-1} 2^{\rho-1}(2 k-1) ! \Gamma(\rho)}{k !(k-2) ! \Gamma(2 k+\rho-1)}\left[t^{2 k+\rho-2} f^{(k-1)}(t)\right]\right]^{(k)}
$$

as a generalization of (5); and proved results corresponding to Widder's for (3) and (4).

In [G] the author discussed the properties of the functions defined by (3) and (4); and by arguments based on the method of steepest descents, obtained independently of Pollard the operator

$$
M_{k t}(f)=\frac{(-)^{k-1} t^{k+\rho-2} \Gamma(2 k+\rho-1) \Gamma(\rho)}{\Gamma(k+\rho)(k-2) !} \sum_{r=0}^{k} \frac{C_{k, r} t^{r} f^{(k+r-1)}(t)}{\Gamma(k+\rho+r-1)}
$$

which has the same effect on (3) and (4) as Pollard's operator $L_{\mathbf{k t}}^{p}$. This was to be expected, since as can easily be verified, the operators (6) and (7) are asymptotically the same for large $k$, and indeed differ only in the outside multipliers.

We shall show in the present work that by certain formal arguments an inversion formula involving a complex integral for (3) and (4) can be obtained from (7). This operator turns out to be a generalization of the one used by Stieltjes [B, pp. 473-476] to invert (2).

2. Definitions and elementary properties. It will be noted that when $\rho$ is not an integer, the nucleus $(x+t)^{-\rho}$ in (3) and (4) is manyvalued. Moreover, even when $\rho$ is an integer, it may be seen from the example

$$
\begin{array}{rlr}
\alpha(t)=0, & 0 \leqq t \leqq c, \\
\alpha(t)=1, & t>c,
\end{array}
$$

that possible singularities of (3) and (4) arise when $t$ is zero or negative. We avoid both these difficulties by considering $f(x)$ in the region $D$, where $D$ is the $x$-plane cut from the origin along the negative real axis. It is open to us to choose any arbitrarily fixed determination for $(x+t)^{-\rho}$ (and indeed for any number $z^{-\rho}$ which occurs in our argu- 
ment), when the nucleus $(x+t)^{-\rho}$ or the function $z^{-\rho}$ will be onevalued and analytic in $D$. For convenience we shall take that determination of $z^{-\rho}$ for which arg $\left[z^{-\rho}\right]$ has its principal value. But it can be seen from subsequent arguments that any fixed determination could have been chosen, and that our results would then be equally valid.

It is convenient at this stage to give some elementary properties of the functions defined by (3) and (4). Proofs have been given by Widder [A] for the case $\rho=1$. The proof in each case is an easy generalization of his methods. We combine them in the following theorem.

THEOREM 2. If the integral in (3) or (4), with the determination of $(x+t)^{-\rho}$ given above, converges for any $x$ in $D$, then it converges for all such $x$, and converges uniformly in any closed subregion of $D$. The functions defined by (3) and (4) are then analytic in $D$, and for (3)

$$
f^{(k)}(x)=\frac{(-)^{k} \Gamma(k+\rho)}{\Gamma(\rho)} \int_{0}^{\infty} \frac{d \alpha(t)}{(x+t)^{k+\rho}}
$$

with a similar result for (4). When (3) converges, $\alpha(t)=o\left(t^{p}\right)$, and when (4) converges $\int_{1}^{t} \phi(u) d u=o\left(t^{p}\right)$, as $t \rightarrow \infty$.

3. Some formal arguments. Widder has shown [H, p. 381] that the operator (5), with variable $t$, can be expressed symbolically as

$$
-\frac{1}{\pi} \sin \pi t D=\frac{1}{2 i \pi}\left[e^{-i \pi t D}-e^{i \pi t D}\right]
$$

where $D=d / d t$, and uses this result to obtain the inversion formula of Stieltjes [B] for the transform (2). We proceed from (7) and write

$$
M_{k t}(f)=\frac{(-)^{k-1} t^{\rho-1} \Gamma(2 k+\rho-1) \Gamma(\rho)}{\Gamma(k+\rho)(k-2) !} \sum_{r=0}^{k} C_{k, r} \frac{t^{k+r-1} D^{k+r-1}}{\Gamma(k+\rho+r-1)} \cdot f(t) .
$$

Using the usual notation $\Theta=t \cdot d / d t$, we see that

$$
\begin{aligned}
M_{k t}(f) & =\frac{t^{\rho-1} \Gamma(2 k+\rho-1) \Gamma(\rho)}{\Gamma(k+\rho)(k-2) !} \sum_{0}^{k} C_{k, r} \frac{(-)^{r}(-\Theta)_{k+r-1}}{\Gamma(k+\rho+r-1)} f(t) \\
& =\frac{t^{\rho-1} \Gamma(2 k+\rho-1) \Gamma(\rho) \Gamma(k-1-\Theta)}{\Gamma(k+\rho)(k-2) ! \Gamma(-\Theta) \Gamma(k+\rho-1)} \sum_{0}^{k} \frac{(-k)_{r}(k-1-\Theta)_{r}}{r !(k+\rho-1)_{r}} f(t) \\
& =\frac{t^{\rho-1} \Gamma(k-1-\Theta) \Gamma(k+\rho+\Theta) \Gamma(\rho)}{\Gamma(-\Theta) \Gamma(\rho+\Theta) \Gamma(k+\rho)(k-2) !} \cdot f(t)
\end{aligned}
$$


on summing the hypergeometric series by Gauss' theorem. Then by Stirling's formula

$$
\lim _{k \rightarrow \infty} M_{k t}=\frac{t^{\rho-1} \Gamma(\rho)}{\Gamma(-\Theta) \Gamma(\rho+\Theta)}
$$

so that we have the formal result

$$
\lim _{k \rightarrow \infty} M_{k t}(f)=\frac{t^{\rho-1} \Gamma(\rho)}{\Gamma(-\Theta) \Gamma(\rho+\Theta)} f(t) .
$$

We express this operation as an integral by the well known formula

$$
\int_{0}^{\pi / 2} \cos ^{p+q-2} \omega \cdot \cos (p-q) \omega d \omega=\pi \Gamma(p+q-1) / 2^{p+q-1} \Gamma(p) \Gamma(q)
$$

[C, p. 40], which is true when $p+q>1$. On setting $p+q=1+\rho$, $p-q=\rho-1+2 \Theta$, the operator in (8) assumes the form

$$
\frac{-(2 t)^{\rho-1} 2 \Theta}{\pi} \int_{0}^{\pi / 2} \cos ^{\rho-1} \omega \cos (\rho-1+2 \Theta) \omega d \omega
$$

$$
=\frac{-(2 t)^{\rho-1} t D}{\pi} \int_{-\pi / 2}^{\pi / 2} \cos ^{\rho-1} \omega e^{i \omega(\rho-1)} \cdot e^{2 i \theta \omega} d \omega .
$$

Now applying (9) to $f(t)=g(u)$, where $t=e^{u}$, and bearing in mind that $e^{a \theta} g(u)=g(u+a)$ formally, we get

$$
\begin{aligned}
\frac{-(2 t)^{\rho} D}{2 \pi} \int_{-\pi / 2}^{\pi / 2} \cos ^{\rho-1} \omega \cdot e^{i(\rho-1) \omega} g(u+2 i \omega) d \omega \\
=\frac{-(2 t)^{\rho} D}{2 \pi} \int_{-\pi / 2}^{\pi / 2} \cos ^{\rho-1} \omega \cdot e^{i(\rho-1) \omega} \cdot f\left(t e^{2 i \omega}\right) d \omega \\
=\frac{-(2 t)^{\rho}}{2 \pi} \int_{-\pi / 2}^{\pi / 2} \cos ^{\rho-1} \omega \cdot e^{i(\rho+1) \omega} f^{\prime}\left(t e^{2 i \omega}\right) d \omega \\
=\frac{-1}{2 i \pi} \int_{C}(z+t)^{\rho-1} f^{\prime}(z) d z,
\end{aligned}
$$

on making the substitution $z=t e^{2 i \omega} . C$ is the circle $|z|=t$, cut at the point $-t$.

We now define as our complex inversion operator

$$
M_{\eta t}(f)=\frac{-1}{2 i \pi} \int_{c_{\eta t}}(z+t)^{\rho-1} f^{\prime}(z) d z,
$$


where $C_{\eta t}$ is the contour which starts at the point $-t-i \eta$, proceeds along the straight line $\operatorname{Im}(z)=-\eta$ to the point $-i \eta$, then along the semi-circle $|z|=\eta, \operatorname{Re}(z) \geqq 0$, to the point $i \eta$, and finally along the line $\operatorname{Im}(z)=\eta$ to the point $-t+i \eta$. Since the integrand in (10) is analytic and one-valued in $D$, the integral along $C$ is equal to the limit as $\eta$ tends to zero of the integral along $C_{\eta t}$.

Before discussing the relation of (10) to (3) and (4), it is of interest to observe that on setting $\rho=1$,

$$
\lim _{\eta \rightarrow 0+} M_{\eta t}(f)=(1 / 2 i \pi)\left[f\left(t e^{-i \pi}\right)-f\left(t e^{i \pi}\right)\right],
$$

which is the inversion operator given by Stieltjes [B, pp. 473-476] for (2).

4. Complex inversion formula. In order to show that (10) will invert (3) and (4) it is desirable to prove three lemmas, which play a decisive part in the inversion theorems. It will be noted that the third of these lemmas gives the value of a generalized form of "Cauchy's singular integral" [D, p. 133 and E, p. 30]. We state the following lemma.

Lemma $4 \mathrm{a}$.

$$
\lim _{\eta \rightarrow 0+} \frac{\eta^{\rho}}{2 i \pi} \int_{0}^{R}\left[\frac{1}{(\eta-i u)^{\rho}}-\frac{1}{(\eta+i u)^{\rho}}\right] \frac{d u}{u}=\frac{1}{2} .
$$

For on setting $u=\eta \tan \phi$, and then making $\eta$ tend to zero through positive values, the expression on the left becomes

$$
\frac{1}{\pi} \int_{0}^{\pi / 2} \frac{\cos ^{\rho-1} \phi \cdot \sin \rho \phi d \phi}{\sin \phi}
$$

and this integral, which clearly converges, is the Cauchy principal value of

$$
\frac{1}{2 i \pi} \int_{-\pi / 2}^{\pi / 2} \frac{e^{i \rho \phi} \cos ^{\rho-1} \phi d \phi}{\sin \phi}
$$

On setting $z=e^{2 i \phi}$, this integral is seen to be equal to

$$
\frac{1}{2 i \pi} \frac{1}{2^{\rho-1}} \int_{c} \frac{(z+1)^{\rho-1} d z}{z-1}
$$

where $C$ is the unit circle indented at $z= \pm 1$, to allow for the pole at $z=1$ and the possible branch point at $z=-1$. Since 
$\lim _{z \rightarrow-1}(z+1)^{p /(z-1)}=0$, and $\lim _{z \rightarrow 1}(z+1)^{p-1}=2^{p-1}$, it follows that the integral has the value $1 / 2$.

LEMMA 4b. If $g(u) \in L(0 \leqq u \leqq R)$ and $g(0+)$ exists, then

$$
\lim _{\eta \rightarrow 0+} \frac{\eta^{\rho}}{2 i \pi} \int_{0}^{R} \frac{g(u)}{u}\left[\frac{1}{(\eta-i u)^{\rho}}-\frac{1}{(\eta+i u)^{\rho}}\right] d u=\frac{1}{2} g(0+) .
$$

For consider

$$
I \equiv \frac{\eta^{\rho}}{2 i \pi} \int_{0}^{R} \frac{g(u)-g(0+)}{u}\left[\frac{1}{(\eta-i u)^{\rho}}-\frac{1}{(\eta+i u)^{\rho}}\right] d u ;
$$

and let a positive $\delta$ be chosen so that $|g(u)-g(0+)|<\epsilon$ for $0<u \leqq \delta$. If the integral in $I$ is taken in two parts, over $(0, \delta)$ and $(\delta, R)$, then

$$
\begin{aligned}
|I(0, \delta)| & \leqq \frac{\epsilon}{\pi} \int_{0}^{\operatorname{src} \tan \delta / \eta} \frac{\cos ^{\rho-1} \phi|\sin \rho \phi| d \phi}{\sin \phi} \text { (on setting } u=\eta \tan \phi \text { ) } \\
& \rightarrow \frac{\epsilon}{\pi} \int_{0}^{\pi / 2} \frac{\cos ^{\rho-1} \phi|\sin \rho \phi| d \phi}{\sin \phi}=\epsilon K,
\end{aligned}
$$

since $\rho$ is positive and $|\sin \rho \phi / \sin \phi|$ is bounded. On the other hand

$$
\begin{aligned}
|I(\delta, R)| & \leqq \frac{\eta^{\rho}}{\pi} \int_{\delta}^{R} \frac{|g(u)-g(0+)|}{u} \frac{d u}{\left(\eta^{2}+u^{2}\right)^{\rho / 2}} \\
& \leqq \frac{\eta^{\rho}}{\pi} \int_{\delta}^{R} \frac{|g(u)-g(0+)| d u}{u^{\rho+1}} .
\end{aligned}
$$

Hence $\lim \sup _{\eta \rightarrow 0+}|I|=0$; and this means the required limit has the value

$$
\lim _{\eta \rightarrow 0+} \frac{g(0+) \cdot \eta^{\rho}}{2 i \pi} \int_{0}^{R}\left[\frac{1}{(\eta-i u)^{\rho}}-\frac{1}{(\eta+i u)^{\rho}}\right] \frac{d u}{u}=\frac{1}{2} g(0+),
$$

by Lemma $4 a$.

LEMMA 4c. If $g(u) \in L(0 \leqq u \leqq R)$, if $0<t<R$, and if $g(t+)$ and $g(t-)$ exist, then

$$
\begin{array}{r}
\lim _{\eta \rightarrow 0+} \frac{\eta^{\rho}}{2 i \pi} \int_{0}^{R} \frac{g(u)}{t-u}\left[\frac{1}{(\eta-i \cdot(t-u))^{\rho}}-\frac{1}{(\eta+i \cdot(t-u))^{\rho}}\right] d u \\
=\frac{1}{2}[g(t+)+g(t-)] .
\end{array}
$$


This is proved by splitting the integral into two parts corresponding to the intervals $(0, t)$ and $(t, R)$, and applying Lemma $4 \mathrm{~b}$ to each part.

We now state and prove the main inversion theorems:

THEOREM 4a. If $\phi(u) \in L(0 \leqq u \leqq R)$ for all positive $R$, and is such that (4) converges, then $\lim _{\eta \rightarrow 0+} M_{\eta t}(f)=[\phi(t+)+\phi(t-)] / 2$ for any positive $t$ at which both $\phi(t+)$ and $\phi(t-)$ exist.

For we have

$$
\begin{aligned}
M_{\eta t}(f) & =\frac{-1}{2 i \pi} \int_{C_{\eta t}}(z+t)^{\rho-1} f^{\prime}(z) d z \\
& =\frac{\rho}{2 i \pi} \int_{C_{\eta t}}(z+t)^{\rho-1} d z \int_{0}^{\infty} \frac{\phi(u) d u}{(z+u)^{\rho+1}} \\
& =\frac{\rho}{2 i \pi} \int_{0}^{\infty} \phi(u) d u \int_{C_{\eta t}} \frac{(z+t)^{\rho-1}}{(z+u)^{\rho-1}} d z \text { (by uniform convergence) } \\
& =\frac{\eta^{\rho}}{2 i \pi} \int_{0}^{\infty} \frac{\phi(u)}{t-u}\left[\frac{1}{(\eta-i \cdot(t-u))^{\rho}}-\frac{1}{(\eta+i \cdot(t-u))^{\rho}}\right] d u .
\end{aligned}
$$

Now let $R>t$, and let the integral be split into two parts corresponding to the intervals $(0, R)$ and $(R, \infty)$. Then by Lemma 3c,

$$
I(0, R) \rightarrow[\phi(t+)+\phi(t-)] / 2
$$

as $\eta \rightarrow 0+$.

Since $I(R, \infty)$ need not converge absolutely, we define $p(t)$ $=\int_{1}^{t} \phi(u) d u$. Then by Theorem $2, p(t)=o\left(t^{p}\right)$ as $t \rightarrow \infty$; and

$$
I(R, \infty)=\frac{-\eta^{\rho} \psi(t, R) p(R)}{2 i \pi \cdot(t-R)}-\frac{\eta^{\rho}}{2 i \pi} \int_{R}^{\infty} p(u) \frac{\partial}{\partial u}\left[\frac{\psi(t, u)}{t-u}\right] d u,
$$

where $\psi(t, u) \equiv[\eta-i(t-u)]^{-\rho}-[\eta+i(t-u)]^{-p}$. The first term clearly tends to zero with $\eta$. If the second be denoted by $I^{\prime}(\eta)$, we have

$$
\begin{aligned}
\left|I^{\prime}(\eta)\right| \leqq & \frac{\eta^{\rho}}{2 \pi} \int_{R}^{\infty} \frac{|p(u)||\psi(t, u)| d u}{(t-u)^{2}} \\
& +\frac{\rho \eta^{\rho}}{2 \pi} \int_{R}^{\infty} \frac{|p(u)|}{u-t}\left|\frac{1}{(\eta-i \cdot(t-u))^{\rho+1}}-\frac{1}{(\eta+i \cdot(t-u))^{\rho+1}}\right| d u .
\end{aligned}
$$

Since $p(u)=o\left(u^{p}\right)$ as $u \rightarrow \infty$, there exists a constant $M$ such that for 
$u \geqq R,|p(u)| \leqq M u^{\rho}$. Then

$$
\begin{aligned}
\left|I^{\prime}(\eta)\right| \leqq & \frac{M \eta^{\rho}}{\pi} \int_{R}^{\infty} \frac{u^{\rho} d u}{(t-u)^{2}\left\{\eta^{2}+(t-u)^{2}\right\} \rho / 2} \\
& +\frac{M \rho \eta^{\rho}}{\pi} \int_{R}^{\infty} \frac{u^{\rho} d u}{(u-t)\left\{\eta^{2}+(t-u)^{2}\right\}(\rho+1) / 2} \\
\leqq & \frac{M \eta^{\rho}(1+\rho)}{\pi} \int_{R}^{\infty} \frac{u^{\rho} d u}{(u-t)^{\rho+2}} .
\end{aligned}
$$

But the integral converges, since $R>t$ and $\rho>0$, so that $\left|I^{\prime}(\eta)\right| \rightarrow 0$. This completes the proof of our theorem.

TheOREM 4b. If (3) converges, then for any positive $t$,

$$
\lim _{\eta \rightarrow 0+} \int_{0}^{t} M_{\eta u}(f) d u=\frac{1}{2}[\alpha(t+)+\alpha(t-)-\alpha(0+)] .
$$

For

$$
\begin{aligned}
\int_{0}^{t} & M_{\eta u}(f) d u \\
= & \frac{-1}{2 i \pi} \int_{0}^{t} d u\left[(z+u)^{\rho-1} f(z) d z-(\rho-1) \int(z+u)^{\rho-2} f(z) d z\right]_{C_{\eta u}} \\
= & \frac{-1}{2 i \pi} \int_{0}^{t}\left[(i \eta)^{\rho-1} f(-u+i \eta)-(-i \eta)^{\rho-1} f(-u-i \eta)\right] d u \\
& +\frac{\rho-1}{2 i \pi} \int_{0}^{t} d u \int_{C_{\eta u}}(z+u)^{\rho-2} f(z) d z .
\end{aligned}
$$

Writing the last term as $I_{1}+I_{2}+I_{3}$, where $I_{1}$ is the integral from $-t-i \eta$ to $-i \eta, I_{2}$ the integral around the semi-circle from $-i \eta$ to $i \eta$, and $I_{3}$ the integral along the straight line from $i \eta$ to $-t+i \eta$, we have

$$
\begin{aligned}
I_{1} & =\frac{\rho-1}{2 i \pi} \int_{0}^{t} d u \int_{0}^{u}(u-\xi-i \eta)^{\rho-2} f(-\xi-i \eta) d \xi \\
& =\frac{\rho-1}{2 i \pi} \int_{0}^{t} f(-\xi-i \eta) d \xi \int_{\xi}^{t}(u-\xi-i \eta)^{\rho-2} d u \\
& \quad \text { (by uniform convergence) } \\
& =\frac{1}{2 i \pi} \int_{0}^{t} f(-\xi-i \eta)\left[(t-\xi-i \eta)^{\rho-1}-(-i \eta)^{\rho-1}\right] d \xi,
\end{aligned}
$$




$$
I_{3}=\frac{-1}{2 i \pi} \int_{0}^{t} f(-\xi+i \eta)\left[(t-\xi+i \eta)^{\rho-1}-(i \eta)^{\rho-1}\right] d \xi
$$

(by a similar argument),

and

$$
\begin{aligned}
I_{2} & =\frac{\rho-1}{2 i \pi} \int_{0}^{t} d u \int_{C_{\eta 0}}(z+u)^{\rho-2} f(z) d z \\
& =\frac{1}{2 i \pi} \int_{C_{\eta 0}}\left[(z+t)^{\rho-1}-z^{\rho-1}\right] f(z) d z .
\end{aligned}
$$

On adding we have

(11) $\int_{0}^{t} M_{\eta u}(f) d u=\frac{1}{2 i \pi} \int_{C_{\eta t}}(z+t)^{\rho-1} f(z) d z-\frac{1}{2 i \pi} \int_{C_{\eta 0}} z^{\rho-1} f(z) d z$.

Now since $\alpha(t)$ is normalized, $f(z)=\rho \int_{0}^{\infty} \alpha(u) d u /(z+u)^{\rho+1}$, and the integral converges uniformly for $z$ on $C_{\eta t}$ and $C_{\eta 0}$. Then the first term in (11) is equal to

$$
\begin{aligned}
& \frac{\rho}{2 i \pi} \int_{C_{\eta t}}(z+t)^{\rho-1} d z \int_{0}^{\infty} \frac{\alpha(u) d u}{(z+u)^{\rho+1}} \\
& \quad=\frac{\rho}{2 i \pi} \int_{0}^{\infty} \alpha(u) d u \int_{C_{\eta t}}\left(\frac{z+t}{z+u}\right)^{\rho-1} \cdot \frac{d z}{(z+u)^{2}} \\
& \quad=\frac{1}{2 i \pi} \int_{0}^{\infty} \frac{\alpha(u) d u}{u-t}\left[\left(\frac{i \eta}{u-t+i \eta}\right)^{\rho}-\left(\frac{-i \eta}{u-t-i \eta}\right)^{\rho}\right] \\
& \quad=\frac{\eta^{\rho}}{2 i \pi} \int_{0}^{\infty} \frac{\alpha(u) d u}{t-u}\left[\frac{1}{(\eta-i \cdot(t-u))^{\rho}}-\frac{1}{(\eta+i \cdot(t-u))^{\rho}}\right]
\end{aligned}
$$

while the second term in (11) is equal to

$$
\begin{aligned}
\frac{-\rho}{2 i \pi} & \int_{C_{\eta 0}} z^{\rho-1} d z \int_{0}^{\infty} \frac{\alpha(u) d u}{(z+u)^{\rho+1}} \\
& =\frac{-\rho}{2 i \pi} \int_{0}^{\infty} \alpha(u) d u \int_{C_{\eta 0}} \frac{z^{\rho-1} d z}{(z+u)^{\rho+1}} \quad \text { (by uniform convergence) } \\
& =\frac{-\eta^{\rho}}{2 i \pi} \int_{0}^{\infty} \frac{\alpha(u) d u}{u}\left[\frac{1}{(\eta-i u)^{\rho}}-\frac{1}{(\eta+i u)^{\rho}}\right]
\end{aligned}
$$

It follows now from Theorem 4 a that $(12) \rightarrow[\alpha(t+)+\alpha(t-)] / 2$ as $\eta \rightarrow 0+$. While if (13) be written as $I(0, R)+I(R, \infty), R$ being posi- 
tive, it follows from Lemma $4 \mathrm{~b}$ that $I(0, R) \rightarrow-\alpha(0+) / 2$, and by an argument similar to that used in the proof of Theorem $4 \mathrm{a}$, it can be seen that $I(R, \infty) \rightarrow 0$ with $\eta$. This completes the proof of the second inversion theorem.

\section{BIBLIOGRAPHY}

A. D. V. Widder, The Stieltjes transform, Trans. Amer. Math. Soc. vol. 43.

B. T. J. Stieltjes, Oeuvres complètes, vol. 2.

C. A. L. Cauchy, Oeuvres complètes, s. 1. vol. 15.

D. A. L. Cauchy, Oeuvres complètes, s. 1, vol. 1.

E. E. C. Titchmarsh, Theory of the Fourier integral, 1937 ed.

F. H. Pollard, Studies on the Stieltjes transform, Dissertation, Harvard, Bull. Amer. Math. Soc. Abstract 48-3-117.

G. D. B. Sumner, The inversion of generalised Stieltjes transforms, Dissertation, Witwatersrand University, 1947.

H. D. V. Widder, The Laplace transform, Princeton University Press.

WitWATERSRAND UNIVERSITY AND

Louisiana State University 